\title{
Influence of nifedipine on interstitial fibrosis in renal transplant allografts treated with cyclosporin A
} T A McCulloch, S J Harper, P K Donnelly, J Moorhouse, P R F Bell, J Walls, J Feehally,
P N Furness
Since its introduction over a decade ago cyclosporin A has been shown to be an effective immunosuppressive agent, with renal allograft survivals better than those on previously available immunosuppressive regimens. ${ }^{12}$ However, nephrotoxicity remains a major problem. This may take two main forms: an acute and reversible rise in serum creatinine; or a chronic, progressive, irreversible decline in renal function, manifest as a steady rise in serum creatinine accompanied by progressive interstitial fibrosis, tubular atrophy, and eventual glomerulosclerosis on biopsy. ${ }^{3-5}$ These histological features are also present in renal biopsy specimens from patients treated with cyclosporin A for reasons other than renal transplantation $^{6-8}$ and an increase in severity with increasing doses of cyclosporin A. ${ }^{9}$ Although many histological features have been suggested as being specific to chronic cyclosporin A toxicity, none has consistently been demonstrated by detailed studies, ${ }^{10}$ with differentiation from chronic rejection being particularly difficult. ${ }^{11}$

The mechanism of chronic cyclosporin A toxicity is controversial but may be due to a constriction of the afferent glomerular arteriole, ${ }^{12-14}$ resulting in chronic low grade ischaemia of the nephron. The histological appearances are consistent with an ischaemic pathogenesis. ${ }^{6}$ Furthermore, mice treated with cyclosporin A can have increased concentrations of collagen type I and IV messenger RNA within the renal interstitium. ${ }^{15}$

Calcium channel blocking drugs are vasodilatory, having an effect on the afferent glomerular arteriole, and in addition, by their effect on voltage dependent slow calcium channels, have a theoretical advantage in ischaemic injury where calcium influx into cells is a major cause of cell damage. ${ }^{16}$ Experimental and clinical data suggest that calcium channel blockade may be effective in reducing acute and chronic cyclosporin $\mathrm{A}$ toxicity. ${ }^{1718}$ This study was designed to investigate the clinical and pathological effects of any possible reduction in cyclosporin $\mathrm{A}$ toxicity by comparison of routinely immunosuppressed renal transplant recipients with those who had also received the calcium channel blocking drug nifedipine since the time of transplant, and to compare both of these groups with patients treated with low dose cyclosporin $\mathrm{A}$, with azathioprine as additional immunosuppression. 
Methods

One hundred and seven consecutive patients undergoing renal allograft transplantation at the Leicester General Hospital from 1989 to 1992 were randomised to one of three immunosuppressive regimens:

Group $A$ : cyclosporin $\mathrm{A}$, initial dose of 17 $\mathrm{mg} / \mathrm{kg}$ per day reduced in a stepwise manner by $2 \mathrm{mg} / \mathrm{kg}$ per week to a maintenance dose of $7 \mathrm{mg} / \mathrm{kg}$ per day at six weeks.

Group B: cyclosporin A as in regimen A plus oral nifedipine retard $10 \mathrm{mg}$ three times daily for one week, then $20 \mathrm{mg}$ twice daily, increasing to $40 \mathrm{mg}$ twice daily, if indicated for hypertension.

Group C: cyclosporin A initial dose $10 \mathrm{mg} / \mathrm{kg}$ per day reducing by $1 \mathrm{mg} / \mathrm{kg}$ per week to a maintenance dose of $4 \mathrm{mg} / \mathrm{kg}$ per day at six weeks. This group received azathioprine at a dose of $1 \mathrm{mg} / \mathrm{kg}$ per day as additional immunosuppression.

Prednisolone was given in an identical regimen in all three groups reducing to a maintenance dose of $10 \mathrm{mg}$ alternate days at six months ( $5 \mathrm{mg}$ daily if diabetic).

Blood pressure control was achieved with agents other than calcium channel blockers in groups $\mathrm{A}$ and $\mathrm{C}$.

Wherever possible, renal allograft biopsy specimens were taken perioperatively (before perfusion) and at one, six, and 12 months after transplantation. Biopsy specimens were taken with a 14 gauge needle under ultrasound guidance and immediately placed in $10 \%$ formalin fixative.

Biopsy specimens were processed in a standard routine manner, cut at $1 \mu \mathrm{m}$, and stained with haematoxylin and eosin. By projecting the slides at a uniform magnification factor on to a wall mounted point matrix a point counting method was used to make a histomorphometric analysis of the interstitial volume fraction in each biopsy specimen. For each biopsy one operator (TAM), unaware of patient details, counted 25 points from each of eight randomly selected fields of cortex (that is, 200 points per biopsy specimen). According to standard morphometric theory, ${ }^{19}$ the proportion of points counted as falling on the interstitium is equal to the volume fraction of the interstitium. This method gave a relative standard error of the measurement for each biopsy specimen of about $10 \%$. A minimum of three glomeruli were considered necessary for an adequate biopsy. Repeat counting of a small proportion of biopsy specimens showed that intraobserver variation was less than $10 \%$ of the estimated interstitial volume percentage. All the biopsy specimens were assessed by a renal histopathologist (PNF), blind to group, for the presence of acute rejection. If present, the biopsy specimen was not included in the analysis as the interstitial oedema, which is part of acute rejection, would influence the measurement of interstitial fibrosis.

Interstitial volume fraction was compared between groups for each of the routine biopsy times (-that is, before perfusion, one month, etc.) by non-paired two-tailed $t$ test.
Numbers of adequate biopsy specimens used in each group

\begin{tabular}{lclcc}
\hline & Group $A$ & Group B & Group C & All \\
\hline Before perfusion (months) & 8 & 18 & 9 & 35 \\
1 & 21 & 26 & 16 & 63 \\
6 & 12 & 15 & 16 & 43 \\
12 & 9 & 20 & 15 & 44 \\
All & 50 & 79 & 56 & 185 \\
\hline
\end{tabular}

At each time of biopsy serum creatinine and glomerular filtration rate $\left(\mathrm{Cr}^{51}\right.$-EDTA clearance) were measured. These results have already been published in detail. ${ }^{20}$ For postperfusion biopsy specimens a correlation was attempted between the interstitial volume fraction (independent variable) of each biopsy specimen and the patient's corresponding glomerular filtration rate (dependent variable) for each group as a whole and for all the biopsy specimens. Possible confounding factors of donor and recipient age, ischaemia, and anastomosis times, HLA mismatch and blood pressure were compared using $\chi^{2}$ or $t$ tests, as appropriate.

\section{Results}

There were no significant differences between the three groups in donor or recipient age, HLA mismatch, ischaemia times, anastomosis times, or mean arterial blood pressure at any time. There was no significant difference in the numbers of documented rejection episodes in the three groups. However, significantly fewer grafts showed delayed primary function in group B than groups A or C $\left(p<0.02 ; \chi^{2}\right) .^{20}$ The total number of graft failures during the study was also smaller in group B than the other groups, but the difference was not significant.

The table shows the numbers of adequate biopsy specimens in each group at each time. Fifteen or more adequate biopsy specimens were obtained at each time interval after transplant in all groups except at six and 12 months in group A where numbers tailed off. The figure shows mean interstitial volume

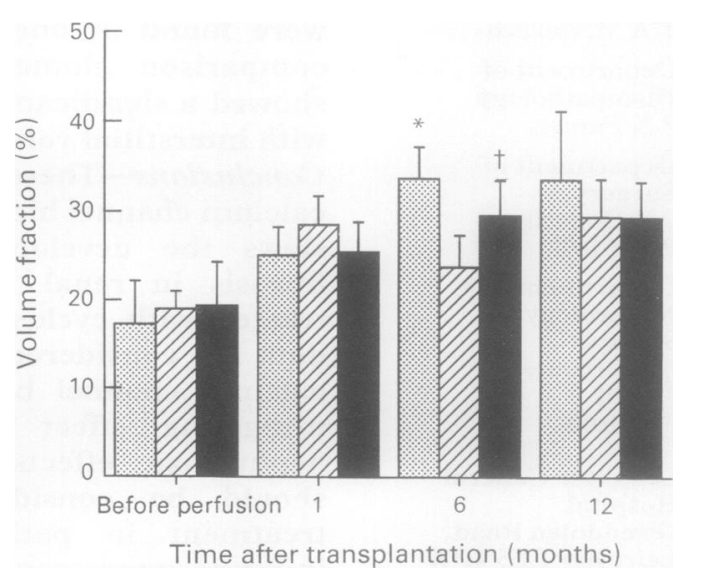

Mean interstitial volume fraction (bars indicate $95 \%$ confidence limits)

圖 = Group $A$. $=$ Group B. $\mathbf{\square}=$ Group $C$.

${ }^{\star}: p<0.001, A v B . \dagger: p<0.05, B v C$. 
fraction as a percentage, with $95 \%$ confidence limits, for each group at each time. The mean interstitial volume fraction increased in time in all groups. No significant differences were present between the groups in the biopsy specimens before perfusion or at one month, but at six months there was significantly less interstitium in group B than groups A $(p<0.001)$ or $C(p<0.05)$, while there was a non-significant difference between groups $\mathrm{A}$ and $\mathrm{C}$.

At 12 months group A still had a higher mean value of interstitium than groups $\mathbf{B}$ or $\mathrm{C}$, but this difference failed to reach significance. Taking all the biopsy specimens at six and 12 months as a single group showed a significant difference between groups $\mathbf{A}$ and $\mathbf{B}$ ( $\mathrm{p}<0.01)$.

Regression of glomerular filtration rate on interstitial volume fraction showed a significant negative correlation in group $A(b=$ $-0.634 \mathrm{ml} /$ minimum per $1.73 \mathrm{~m}^{2}$ per $1 \%$ increase in interstitial volume, $r=0.399, p<$ 0.05 ), and when all 150 post-perfusion biopsy specimens were taken into account $(b=$ $-0.402 \mathrm{ml} / \mathrm{minimum}$ per $1.73 \mathrm{~m}^{2}$ per $1 \%$ increase in interstitial volume, $r=-0.245$, $\mathrm{p}<0.01)$. Glomerular filtration rate did not correlate significantly with interstitial volume fraction in groups B or C alone or when biopsy specimens from all groups at any one time point were analysed together.

\section{Discussion}

In the present study interstitial volume fraction was significantly smaller in the group treated with nifedipine than in those receiving the same cyclosporin A regimen without calcium channel blockade at six months. At this point the mean interstitial volume fraction value in the triple treatment group was between that of the other two groups, reaching significance when compared with group B but not group A. Although group A had a higher mean interstitial volume at 12 months than either groups B or C, the difference did not reach significance and the values for groups $B$ and $C$ were very similar. The absence of the significant difference between groups A and B at 12 months may result from the small numbers of biopsy specimens available for assessment in group A at 12 months. Blood pressure control could not be achieved in some patients without calcium channel blocking agents, thus removing them from the protocol. There were also more graft failures in group A than group B: failed grafts are not biopsied, removing from the analysis a group of cases in which high values for interstitial volume fraction would be expected. Adding the six and 12 month biopsy specimens together to increase numbers showed retention of the significant difference between groups $\mathrm{A}$ and $\mathrm{B}$. Why a difference between the interstitial volume fractions in groups B and $C$ should be present at six months but not at 12 months is not clear.

The clinical results correlated well with the morphometric findings. Group B showed sig- nificantly lower serum creatinine than group A at one, six, and 12 months, while glomerular filtration rate was significantly higher in both groups $B$ and $C$ than group $A$ at six and 12 months. ${ }^{20}$

In previous morphometric studies Ruiz et $a l^{21}$ showed an increase in interstitial volume fraction in patients treated with cyclosporin A compared with those who were not, however, as in the present study, this increase was only apparent in biopsy specimens taken six months or more after transplantation. Wilczek el $a l^{22}$ showed an increase in interstitial volume fraction, at two years after transplantation, in biopsy specimens from patients treated with high dose cyclosporin A compared with those on lower doses, analogous to the present comparison of groups A and C. Both authors have stressed the need to exclude biopsy specimens showing acute rejection as any histomorphometric technique used only gives an estimate of interstitial volume fraction and not fibrosis specifically, and before this was taken into account no differences existed. Notably, semiquantitative methods (even within the same studies) were not able to demonstrate the same differences, ${ }^{21-23}$ underlining the greater sensitivity of histomorphometry. The effect of nifedipine on cyclosporin $\mathrm{A}$ induced renal interstitial fibrosis has never been measured before.

When directly compared, the glomerular filtration rate showed a significant negative correlation with interstitial volume fraction when all post-perfusion biopsy specimens were considered and when those from group A were considered alone-that is, as interstitial volume increased, glomerular filtration rate decreased. The absence of an analogous correlation in groups B and C when each was considered alone may be a reflection of their relatively lower overall values of interstitial volume fraction, in that any correlation is weak until relatively high interstitial volume fraction values are reached or that the correlation is non-linear. The relatively weak correlation in this study also contrasts with that seen between glomerular filtration rate and severity of interstitial change in native kidneys with predominantly glomerular disease, where interstitial change, as opposed to glomerular or vascular change, was much the strongest predictor of glomerular filtration rate. ${ }^{24}$ It seems reasonable to suggest that in relatively healthy kidneys this correlation is difficult to demonstrate.

In conclusion, the present histomorphometrically based findings, considered together with data on graft function, strongly support the hypothesis that calcium channel blockade may help to reduce medium to long term cyclosporin nephrotoxicity.

We thank the technical staff of the Department of We thank the technical staff of the Department of
Histopathology, Leicester General Hospital, for their help in Histopathology, Leicester General Hospital, for their help in
providing the processed histological material used in the study. providing the processed histological material used in the study. SJH is a Wellcome Trust
Grant No 034937/91.

1 European Multicentre Transplant Trial. Cyclosporine in renal cadaveric transplantation: 5 year follow-up of a multicentre trial. Lancet 1987;ii:506-7. 
2 Canadian Multicentre Transplant Study Group. A randomised clinical trial of cyclosporine in cadaveric renal transplantation at three years. $N$ Engl $f$ Med 1986 314:1219-25.

3 Myers BD. Cyclosporine nephrotoxicity. Kidney Int 1986; 30:964-74.

4 Bignardi G, Nield GH, Hartley RB, et al. Histopathological changes in cyclosporine-treated renal allografts at one and twelve months. Nephrol Dial Transplant 1987;2:366-70.

5 Savoldi S, Scolari F, Sandrini S, et al. Cyclosporine chronic nephrotoxicity: histologic follow-up at six and eighteen months after renal transplantation. Transplant eighteen months after renal tran
Proc 1988;20(suppl 3):777-84.

6 Myers BD, Newton L, Boshkos C, Luetscher E, Perlroth M. Cyclosporine associated chronic nephropathy. $N$ Engl f Med 1984;311:699-729.

7 Svenson K, Bohman S-O, Hallgren R. Renal interstitial fibrosis and vascular changes. Occurrence in patient with autoimmune diseases treated with Cyclosporine. Arch Intern Med 1986;146:2007-10.

8 Palestine AG, Austin HA, Balow JE, et al. Renal histopathologic alterations in patients treated with Cyclosporine for uveitis. $N$ Engl 7 Med 1986; 314:1293-8.

9 Dieterle A, Gratwohl A, Nizze $\mathrm{H}$, et al. Chronic cyclosporine-associated nephrotoxicity in bone marrow cyclosporine-associated nephrotoxicity in bone marrow

10 d'Ardenne AJ, Dunnill MS, Wood RFM. Cyclosporine treatment does not cause specific histologic changes in human renal allografts. Transplant Proc 1985:15:2821.

11 Nield GH, Taube DH, Hartley RB, et al. Morphological differentiation between rejection and cyclosporine nephrotoxicity in renal allografts. $f$ Clin Pathol 1986; 39:152-9.

12 Gladue RP, Newborg MF. The protective effect of the thromboxane synthetase inhibitor dazmegrel on nephrotoxicity in cyclosporine treated rats. Transplantation 1991;52:837-41.

13 Petric R, Freeman D, Wallace C, McDonald J, Stiller C, Keown P. Modulation of experimental nephrotoxicity by
inhibition of thromboxane synthesis. Transplantation inhibition of thro

14 Bunke M, Wilder L, Martin A. The effect of cyclosporin on agonist-stimulated glomerular and mesangial cel vasodilatory prostaglandin production. Transplantation 1991;52:717-22.

15 Wolf G, Nielson EG. Increases in the levels of collagen types I and IV messenger ribonucleic acid in murine kidneys after treatment with cyclosporin. Nephron 1992;60:87-91.

16 Cheung JY, Bonventre JV, Malis CD, Leaf A. Calcium and ischaemic injury. $N$ Engl 7 Med 1986;314:1670-6.

17 McNally PG, Baker F, Mistry N. Effect of nifedipine on renal haemodynamics in an animal model of cyclosporin renal haemodynamics in an animal model of

18 McNally PG, Walls J, Feehally J. The effect of nifedipine on renal function in normotensive cyclosporin A-treated on renal function in normotensive cyclosporin A-treated
renal allograft recipients. Nephrol Dial Transplant 1990; 5:962-8.

19 Aherne WA, Dunnil MS. Morphometry. London: Edward Arnold, 1982 .

20 Harper SJ, Moorhouse J, Veitch PS, et al. Nifedipine improves immediate and 6 and 12 month graft function in cyclosporin A (Cy A) treated renal allograft recipients. Transplant Int 1992;5(suppl 1):S69-72.

21 Ruiz P, Kolbeck PC, Scroggs MW, Sanfilippo F Associations between Cyclosporine therapy and interstiAssociations between Cyclosporine therapy and intersti-
tial fibrosis in renal-allograft biopsies. Transplantation tial fibrosis in

22 Wilczek HE, Groth CG, Bohman S-O. Effect of reduced cyclosporine dosage on long-term renal allograft histology. Transplant Int 1992;5:65-70.

23 Santelli G, Hiesse C, Schovaert D, et al. Pathology of two year renal biopsies in cyclosporine and conventionally immunosuppressed renal transplants. Transplant Proc 1989;21:1674-6.

24 Schainuck LI, Striker GE, Cutler RE, Banditt EP. Structural-functional correlations in renal disease. Part II: the correlations. Hum Pathol 1970;1:631-41. 